\title{
BAG3 promotes the phenotypic transformation of primary rat vascular smooth muscle cells via TRAIL
}

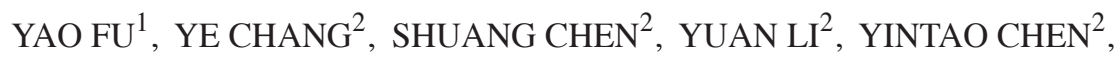 \\ GUOZHE SUN ${ }^{2}$, SHASHA YU ${ }^{2}$, NING YE ${ }^{2}, \mathrm{CHAO} \mathrm{LI}^{3}$ and YINGXIAN SUN ${ }^{2}$ \\ ${ }^{1}$ Department of Cardiology, Shenjing Hospital of China Medical University, Shenyang, Liaoning 110004; \\ ${ }^{2}$ Department of Cardiology, The First Hospital of China Medical University, Shenyang, Liaoning 110001; ${ }^{3}$ Department \\ of Biochemistry and Molecular Biology, China Medical University, Shenyang, Liaoning 110122, P.R. China
}

Received October 12, 2016; Accepted February 8, 2018

DOI: $10.3892 /$ ijmm.2018.3493

\begin{abstract}
Under normal physiological condition, the mature vascular smooth muscle cells (VSMCs) show differentiated phenotype. In response to various environmental stimuluses, VSMCs convert from the differentiated phenotype to dedifferentiated phenotype characterized by the increased ability of proliferation/migration and the reduction of contractile ability. The phenotypic transformation of VSMCs played an important role in atherosclerosis. Both Bcl-2-associated athanogene 3 (BAG3) and tumor necrosis factor-related apoptosis inducing ligand (TRAIL) involved in apoptosis. The relationship between BAG3 and TRAIL and their effects the proliferation and migration in VSMCs are rarely reported. This study investigated the effects of BAG3 on the phenotypic modulation and the potential underlying mechanisms in primary rat VSMCs. Primary rat VSMCs were extracted and cultured in vitro. Cell proliferation was detected by cell counting, real-time cell analyzer (RTCA) and EdU incorporation. Cell migration was detected by wound healing, Transwell and RTCA. BAG3 and TRAIL were detected using real-time PCR and western blotting and the secreted proteins in the cultured media by dot blot. The expression of BAG3 increased with continued passages in cultured primary VSMCs. BAG3 promoted the proliferation and migration of primary rat VSMC in a time-dependent manner. BAG3 significantly increased the expression of TRAIL while had no effects on its receptors. TRAIL knockdown or blocking by neutralizing antibody inhibited the proliferation of VSMCs induced by BAG3. TRAIL knockdown exerted no obvious influence on the migration of VSMCs. Based on this study, we report for
\end{abstract}

Correspondence to: Dr Yingxian Sun, Department of Cardiology, The First Hospital of China Medical University, 155 Nanjing North Street, Heping, Shenyang, Liaoning 110001, P.R. China

E-mail: yxsun@mail.cmu.edu.cn

Key words: Bcl-2-associated athanogene 3, vascular smooth muscle cells, proliferation, migration, tumor necrosis factor-related apoptosis inducing ligand the first time that BAG3 was expressed in cultured primary rat VSMCs and the expression of BAG3 increased with continued passages. Furthermore, BAG3 promoted the proliferation of VSMCs via increasing the expression of TRAIL. In addition, we also demonstrated that BAG3 promoted the migration of VSMCs independent of TRAIL upregulation.

\section{Introduction}

The formation of atherosclerotic plaque is the pathological basis of coronary heart disease (CHD), vascular complication of hypertension and restenosis after angioplasty or bypass (1). The phenotypic transition of vascular smooth muscle cells (VSMCs) plays an important role in the formation and development of atherosclerosis (AS) (1). Under normal physiological condition, the mature VSMCs show differentiated phenotype, and mainly perform contractile function and the proliferation and migration are inefficient (2). They can synthezise and express some contractile markers specific to smooth muscle, including $\alpha$-SMA, SM $22 \alpha$ and $\alpha$-SM-actin (3). Unlike most mature cells such as skeletal muscle cells and cardiomyocytes, VSMCs are not terminally differentiated cells and have remarkable plasticity $(2,4)$. VSMCs were able to convert from the differentiated phenotype to dedifferentiated phenotype which can ensure rapid adaptation to various environmental stimuli (4). The dedifferentiated phenotype was characterized by the increased ability of proliferation/migration and the reduction of contractile ability (5). Though an increasing number of studies have reported various factors and mechanisms that may control VSMCs phenotype modulation, further efforts are still required to elucidate the detailed mechanism and biological importance of the phenotype modulation of VSMCs.

Bcl-2-associated athanogene 3 (BAG3) is a member of BAG family and plays an important role in diverse cellular behaviors including cell apoptosis, autophagy, proliferation, adhesion, migration and differentiation (6-9). Accumulating evidence indicates that BAG3 is associated with various cardiovascular diseases such as myocardial hypertrophy, dilated cardiomyopathy, Takotsubo cardiomyopathy and chronic heart failure (10-13). Normal tissues seldom express BAG3, except for cardiomyocytes and skeletal muscle cells, but its expression is induced upon exposure to various stressful 
stimuli (14). However, there is no previous investigation of BAG3 function in the phenotype modulation of VSMCs.

Tumor necrosis factor-related apoptosis inducing ligand (TRAIL), a member of tumor necrosis factor (TNF) family, belongs to type II membrane protein (15). TRAIL can interact with five different receptors, DR4 (TRAIL-R1), DR45 (TRAIL-R2), DcR1 (TRAIL-R3), DcR2 (TRAIL-R4), and OPG. After binding with death receptors DR4 and DR5, TRAIL can trigger cell apoptosis. Due to lack of the intracellular death domain, DcR1 and DcR2 are considered as decoy receptors to protect normal cells from apoptosis. OPG can work in bone tissue metabolism (16-18). Chiappetta et al reported that BAG3 could downmodulate the apoptotic response to TRAIL in human neoplastic thyroid cells (19). However, the relationship between BAG3 and TRAIL and their effects the proliferation and migration in VSMCs are rarely reported.

The present study analyzed BAG3 expression in VSMCs and investigated its role in the phenotypic transition of VSMCs. Moreover, we determined whether BAG3 could promote the proliferation and migration of VSMCs via TRAIL.

\section{Materials and methods}

Ethics statement. Animals used in our study were treated in accordance with the National Institutes of Health (NIH) Guide for the Care and Use of Laboratory Animals. The procedures was in accordance with the ethical standards of the Committee on Animal Experimentation of China Medical University (project identification code, SCXK-2013-0001).

Materials. Real-Time PCR system was purchased from Applied Biosystems (Foster City, CA, USA). Click-iT Nascent RNA Capture kit was purchased from Invitrogen Life Technologies (Carlsbad, CA, USA). Western blotting related equipment was purchased from Invitrogen Life Technologies. EdU Alexa Fluor 555 Imaging kit was purchased from Invitrogen Life Technologies ${ }^{\text {TM }}$. The real-time cell analyzer (RTCA) xCELLigence system was purchased from ACEA Biosciences (San Diego, CA, USA). Fluorescence microscope (CKX41-F32FL) was purchased from Olympus (Tokyo, Japan). MicroChemi 4.2 was purchased from DNR Bio-Imaging Systems, Ltd. (Jerusalem, Israel). Smart Blotter SB-10 was purchased from Wealtech (Reno, NV, USA). Transwell related equipment was purchased from BD Biosciences (Franklin Lakes, NJ, USA). Microplate reader was purchased from Bio-Rad Laboratories, Inc. (Hercules, CA, USA). Antibodies for $\alpha$-SM-actin, BAG3, DcR1, DcR2, DR4, DR5, OPG and glyceraldehyde 3-phosphate dehydrogenase (GAPDH) were all purchased from Santa Cruz Biotechnology, Inc. (Santa Cruz, CA, USA). Lentiviral vectors were purchased from GeneChem (Shanghai, China).

Isolation and culture of primary VSMCs. Neonatal rats 1-2 days old were sacrificed by cervical dislocation and disinfected with $75 \%$ alcohol, then moved into the clean bench. The thoracic aorta was excised and the inner/outer layers of blood vessel were removed. And then primary neonatal rat VSMCs were isolated as previous described by us (20). VSMCs were cultured in complete medium including $10 \%$ fetal bovine serum (FBS) and penicillin-streptomycin (100 U/ml-100 $\mu \mathrm{g} / \mathrm{ml})$ at $37^{\circ} \mathrm{C}, 5 \% \mathrm{CO}_{2}$, and with humidified atmosphere as previously described $(20,21)$. Media were changed every other day. After primary cells reached $80-90 \%$ confluence, $0.25 \%$ trypsin was added into the culture plate for digestion. Thirty seconds later, serum-containing medium was used to terminate the digestion. Subsequently, a part of the cells were moved into a new culture dish. After attachment, cells were incubated with complete medium, which was passage 2 of VSMCs. With these methods, cells between passages 1-8 were obtained and applied for the next experiments.

Transduction of BAG3 and TRAIL to primary rat VSMCs using lentiviral vectors. The lentiviral plasmids labelled by green fluorescent protein (GFP) were used in the knockdown experiments, which contained short hairpin RNA (shRNA) against rat TRAIL or control shRNA. There were five shRNA oligonucleotides specific for rat TRAIL, i.e., TRAIL \#1, 2, 3, 4 and 5. Furthermore, the lentiviral plasmids labelled by GFP containing BAG3 cDNA were used in the overexpression experiments. Transfection of shRNA oligonucleotide was performed with Lipofectamine 2000 (Invitrogen Life Technologies) according to the manufacturer's recommendations. Transduced cells were cultured for 2 days and then proteins were extracted and analyzed by western blotting.

Cell proliferation assay. Cells were seeded into 6-well plates at a density of $1 \times 10^{4}$ cells/well and cultured with $10 \%$ FBS. The medium was changed every 2 days or as necessary. Cell number at the indicated time-point was determined by counting using a haemocytometer.

Immunofluorescence. Cells were fixed with $4 \%$ paraformaldehyde for $15 \mathrm{~min}$ at room temperature. After washed with phosphate-buffered saline (PBS) three times, cells were permeabilized with $0.25 \%$ Triton $\mathrm{X}-100$ in PBS for $5 \mathrm{~min}$. After blocked with $1 \%$ BSA, cells were incubated with first antibody (1:200) in PBS overnight at $4^{\circ} \mathrm{C}$. After washed with PBS three times, cells were incubated with secondary antibody for $1 \mathrm{~h}$ in the dark. After washed with PBS three times, cells were incubated with $0.1 \%$ DAPI for $5 \mathrm{~min}$ at room temperature. Finally cells were analyzed under a fluorescent microscope.

Western blotting. Western blotting was performed as our previous studies indicated $(20,22)$. Briefly, cells were solubilized in a lysis buffer for $30 \mathrm{~min}$, and then total protein concentrations were measured by a BCA Protein Assay kit. After heat denaturation, the samples were analyzed on a 12 or 14\% Tris-glycine gradient gel, and then transferred to PVDF membranes and blocked with 5\% non-fat milk in Tris-buffered saline (TBS) for $1.5 \mathrm{~h}$ at the room temperature. The membranes were incubated with primary antibody overnight at $4^{\circ} \mathrm{C}$. After washing three times with TBS, the membranes were incubated with secondary antibodies for $1.5 \mathrm{~h}$ at room temperature. After washing steps, immunoreactive binding were detected with enhanced chemiluminescence (ECL) (Amersham Biosciences, Piscataway, NJ, USA). The band intensity was quantified using ImageJ 1.47 software and GAPDH as a control.

Dot blot. The secreted proteins in the media were extracted and quantified similarly to those in western blotting. Then $100 \mu \mathrm{l}$ 
sample volume was blotted onto a nitrocellulose membrane and blocked with $5 \%$ non-fat milk for $1.5 \mathrm{~h}$ at the room temperature. Subsequently, the membranes were incubated with first antibody at 1:1,000 dilution overnight at $4^{\circ} \mathrm{C}$. After washing three times with TBS, the membranes were incubated with secondary antibodies for $1 \mathrm{~h}$ at room temperature. After washing three times with TBS, immunoreactive binding was detected with ECL detection reagent with MicroChemi 4.2.

MTT assay. MTT assay was used to determined cell viability. VSMCs were seeded in a 96-well plate at a density of $4 \times 10^{3}$ cells/well. After cultured $48 \mathrm{~h}$, cells were incubated with MTT solution (final concentration, $5 \mathrm{mg} / \mathrm{ml}$ ) for $4 \mathrm{~h}$ at $37^{\circ} \mathrm{C}$. Then the culture media containing MTT were removed and replaced with $100 \mu 1$ DMSO. Then the plate was gently rotated on a linear and orbital shaker for $5 \mathrm{~min}$ to completely dissolve the precipitation. The absorbance was measured with microplate reader at $570 \mathrm{~nm}$. The percentage of cell viability was calculated according to the following formula: cell viability $(\%)=$ optical density $(\mathrm{OD})$ of the treatment group/OD of the control group $\times 100 \%$.

RTCA proliferation assays. To analyze the cell proliferation continuously over time, growth curve assays were performed in RTCA in quadruplicate with the xCELLigence system according to the methods described (23). Briefly, 5,000 cells/ well were seeded in RTCA E-plates. After the chambers were set up, the RTCA E-plate was put into xCELLigence instrument at $37^{\circ} \mathrm{C}, 5 \% \mathrm{CO}_{2}$ incubator and cell index was recorded every $15 \mathrm{~min}$. The shift of the electrical impedance was expressed as the cell index, which was a parameter of cell viability.

EdU incorporation analysis. As described by our previous studies $(20,24)$ and the manufacturer's instructions, DNA synthesis rate in VSMCs was determined by EdU incorporation analysis using Click-iT ${ }^{\text {тм }}$ EdU Alexa Fluor 555 Imaging kit. Briefly, the cells were incubated with EdU-labeling solution for $8 \mathrm{~h}$ at $37^{\circ} \mathrm{C}$, and then fixed with $4 \%$ cold formaldehyde for $30 \mathrm{~min}$ at room temperature. After permeabilization with $1 \%$ Triton X-100, the cells were reacted with Click-iT reaction cocktails (Invitrogen Life Technologies) for $30 \mathrm{~min}$. Subsequently, the DNA contents of the cells were stained with Hoechst 33342 for 30 min. Finally, EdU-labeled cells were counted using ImageJ 1.47 software and normalized to the total number of Hoechst-stained cells. At least 500 cells in each experiment were counted, EdU-positive cells were expressed as a percentage of the total cells.

Wound healing assay. Cells at 80-90\% confluence were wounded with a $200 \mu \mathrm{l}$ pipette tip and incubated with SFM or $\mathrm{CM}$ for $24 \mathrm{~h}$. Multiple views of the leading edge of the scratch were photographed under a microscope at 0 and $24 \mathrm{~h}$. The experiments were performed three times independently.

Transwell migration assay. For the Transwell migration assay (8- $\mu \mathrm{m}$ pores; BD Biosciences), cells were seeded at density of $2 \times 10^{6}$ cells in the upper chamber. The lower chamber was filled with SFM or CM. After cultured for $24 \mathrm{~h}$, cells on the upper chamber were removed by gentle abrasion with a cotton bud, and the cells on the lower chamber were fixed and stained with Hoechst 33342. Three experiments were performed independently. The cells that passed through the filter were photographed under fluorescence microscope with ultraviolet light. Hoechst-labeled cells in five representative microscopic fields were counted using ImageJ 1.47 software.

RNA extraction and real-time ( $R T)-P C R$. The total RNA was extracted by Qiagen RNeasy Mini kit (Qiagen, Berlin, Germany). After the determination of concentration, the synthesis of cDNA was performed. With the primer design software we synthesized the sense and antisense primers of each fragment: TRAIL sense primer, 5'-tcgtgatcttcacagtgctcc tgcagtc-3' and antisense primer, 5'-tctaacgagctgacggagttgcca cttg-3'; DcR1 sense primer, 5'-gattacaccaacgcttccaac-3' and antisense primer, 5'-gctggtgttcattgtctcttc-3'; DcR2 sense primer, 5'-ttcttgcetgctatgtacag-3' and antisense primer, 5'-agga tggtggtcactgtctc-3'; DR4 sense primer, 5'-gagtacatctaggtgcgtt cctgg-3' and antisense primer, 5'-agagccccacactttgctgg-3'; DR5 sense primer, 5'-tagcactcactggaatgacc-3' and antisense primer, 5'-gtggacacattcgatgtcac-3'; OPG sense primer, 5'-gcctaactggc ttagtgtcttg-3' and antisense primer, 5'-ccaatgtgccgctgcagctg-3'; GAPDH sense primer, 5'-cgtcccgtagacaaaatggt-3' and antisense primer, 5'-ttgatgttagtggggtctcg-3'. Quantitative RT-PCR was run and analyzed with the 7500 RT-PCR system (Applied Biosystems). Results were normalized against those of GAPDH and presented as arbitrary unit.

Label and capture nascent RNA. Click-iT Nascent RNA Capture kit was used to detect newly synthesized RNA according to the manufacturer's instructions. 5-Ethymyl uridine (EU) is an alkyne-modified uridine analog and it could be efficiently and naturally incorporated into the nascent RNA. Cells were incubated in $0.2 \mathrm{mM}$ of EU for $4 \mathrm{~h}$ and total RNA labeled with EU was isolated using TRIzol reagent (Invitrogen Life Technologies). Then EU-labeled RNA was biotinylated in a Click-iT reaction buffer with $0.5 \mathrm{mM}$ of biotin azide and subsequently captured on streptavidin magnetic beads.

Statistical analysis. All data were obtained from at least three individual experiments. Continuous variables were expressed as the mean $\pm \mathrm{SD}$ and tested by one-way analysis of variance (ANOVA) or Student's t-test. Categorical variables were expressed as percentage and tested by Chi-square test. All the statistical analyses were performed using SPSS statistical software for Windows, version 17.0 (SPSS, Inc., Chicago, IL, USA) and P-values $<0.05$ were considered statistically significant.

\section{Results}

The expression of BAG3 increases with continued passages in cultured primary VSMCs. BAG3 is rarely expressed in primary VSMCs, which were mainly in contractile phenotype characterized by expression of $\alpha$-SM-actin. We also found that BAG3 expression was negatively related with VSMC marker protein $\alpha$-SM-actin. BAG3 expression increased and $\alpha$-SM-actin expression decreased with continued passages in cultured primary VSMCs (Fig. 1A and B). This was furthermore confirmed by the results of immunofluorescence staining (Fig. 1C). Passage 1 (differentiated phenotype) and passage 5 (undifferentiated phenotype) were selected to 
A

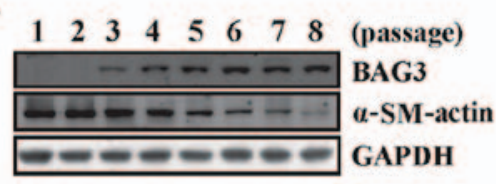

B

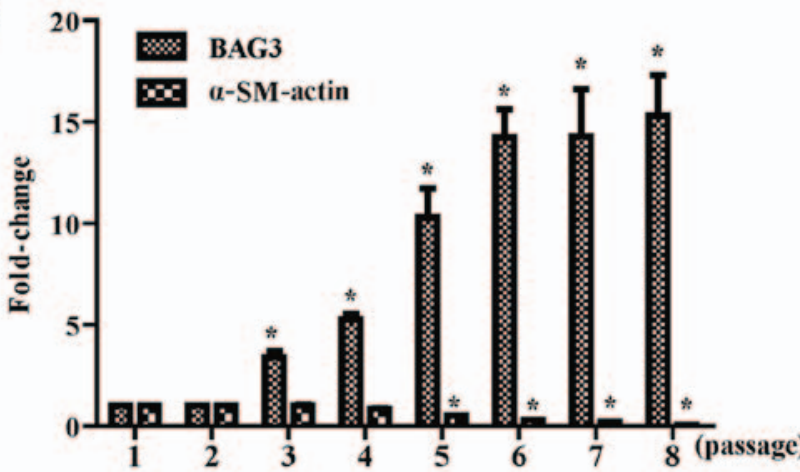

C

BAG3
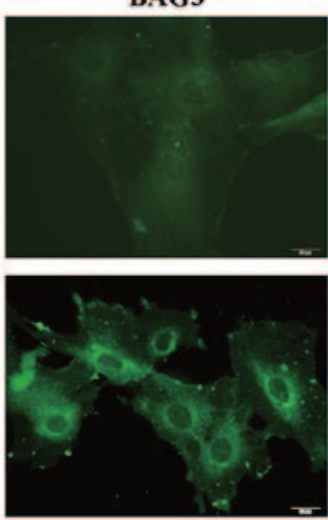

a-SM-actin
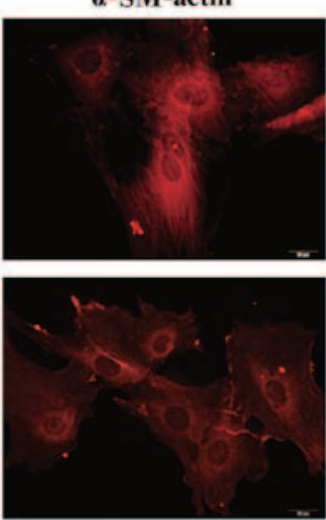

DAPI
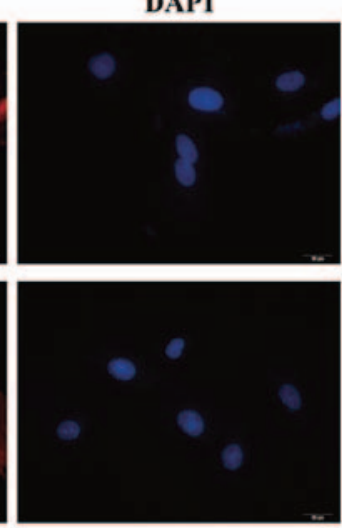
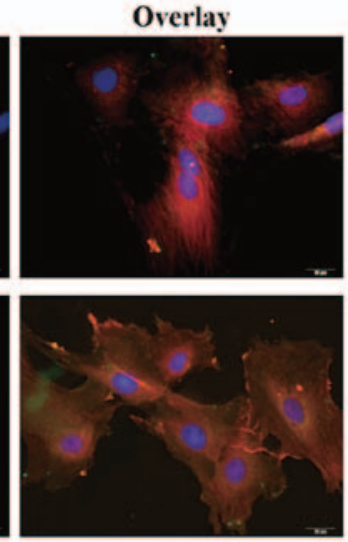

passage 1

passage 5

Figure 1. The expression of Bcl-2-associated athanogene 3 (BAG3) increased with continued passages in cultured primary rat vascular smooth muscle cells (VSMCs). Primary rat VSMCs were cultured in vitro and passages 1-8 were collected. The expression of BAG3 and $\alpha$-SM-actin in passages 1-8 were detected by (A) western blotting and (B) quantified by ImageJ, GAPDH served as a control. (C) The protein expression of BAG3 and $\alpha$-SM-actin in passages 1 and 5 were determined by immunofluorescence assay. The experiments were all repeated three times with reproducible results. "p $<0.05$ compared with the control.

conduct the immunofluorescence staining assay. The fluorescence intensity of BAG3 increased observably in VSMCs of passage 5 compared with that in passage 1 (Fig. 1C). On the contrary, the fluorescence intensity of $\alpha$-SM-actin decreased observably in VSMCs of passage 5 compared with that in passage 1 (Fig. 1C).

BAG3 promotes the proliferation of primary rat VSMCs. We have generated lentiviral vectors to overexpress BAG3 in order to explore the role of BAG3 in proliferation of primary rat VSMCs. Measurement of $\mathrm{GFP}^{+}$cells under fluorescence microscopy demonstrated that transduction efficiency by lentiviral vectors at 100 multiplicity of infection (MOI) was $80-90 \%$ (Fig. 2A). Western blotting confirmed that BAG3 was successfully expressed in VSMCs infected with BAG3-containg lentivirus (Fig. 2B and C). Importantly, results from cell counting (Fig. 2D), RTCA (Fig. 2E) and EdU staining (Fig. 2F) consistently demonstrated that forced overexpression of BAG3 could significantly promote the proliferation of primary rat VSMCs in both SFM group (serum free medium) and CM group (complete medium with 10\% FBS).

BAG3 promotes the migration of primary rat VSMCs. Wound healing assay showed that BAG3 significantly increased the migration of primary rat VSMCs at $24 \mathrm{~h}$ compared with control group (Fig. 3A). Transwell assay demonstrated that the number of invaded cells in BAG3-overexpression VSMCs group was significantly higher than those in the control group (Fig. 3B and C). RTCA also confirmed that BAG3 promoted migration of primary rat VSMCs in a time-dependent manner (Fig. 3D).

TRAIL was implicated in the proliferation mediated by BAG3 in primary rat VSMCs. We wanted to clarify the mechanism underlying the proliferation and migration of primary rat VSMCs promoted by BAG3 overexpression. The results of RT-PCR demonstrated that BAG3 overexpression could significantly increase the mRNA level of TRAIL, but had no obvious effects on the receptors of TRAIL including DcR1, DcR2, DR4, DR5 and OPG (Fig. 4A). The increase in mRNA level could result from the increase of mRNA synthesis or decrease of mRNA degradation. Next, TRAIL gene transcription was analyzed using Click-iT Nascent RNA Capture kit to label and isolate newly synthesized RNA. The results of RT-PCR demonstrated that BAG3-overexpression did not alter the level of newly synthesized TRAIL mRNA (Fig. 4B). Consistently, western blotting demonstrated that BAG3 overexpression could promote the expression of TRAIL protein while have no effects on the receptors of TRAIL (Fig. 4C and D). Furthermore, dot blot demonstrated that BAG3-overexpression could increase the level of secreted TRAIL protein in the cultured media (Fig. 4E).

BAG3 promotes the proliferation of primary rat VSMCs via TRAIL. To investigate the potential involvement of TRAIL in the proliferation of VSMCs induced by BAG3, a neutralizing antibody against TRAIL (TRAIL Ab) was used. Cells 

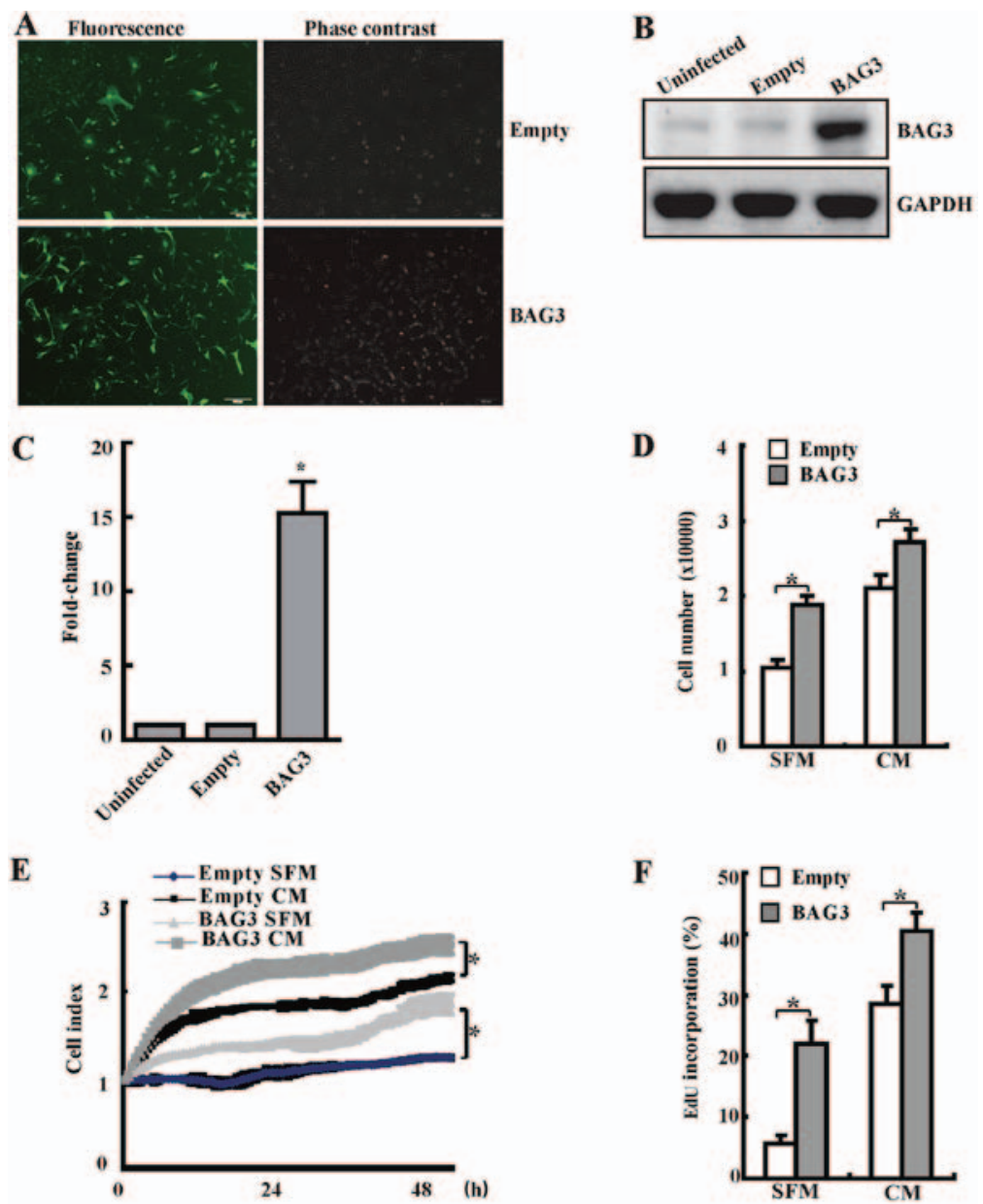

Figure 2. Effects of Bcl-2-associated athanogene 3 (BAG3) on the proliferation of primary rat vascular smooth muscle cells (VSMCs). (A) We have generated lentiviral vectors to overexpress BAG3 in primary rat VSMCs. (B) The protein expression of BAG3 was detected by western blotting and (C) quantified by ImageJ, GAPDH served as a control. (D) Cell proliferation was determined by cell counting and (E) real-time cell analyzer (RTCA). (F) Cell proliferation was further confirmed by EdU staining and EdU incorporation was calculated as EdU ${ }^{+}$cells/total cells and quantified by ImageJ. The experiments were all repeated three times with reproducible results. ${ }^{\mathrm{p}} \mathrm{p}<0.05$ compared with the control.
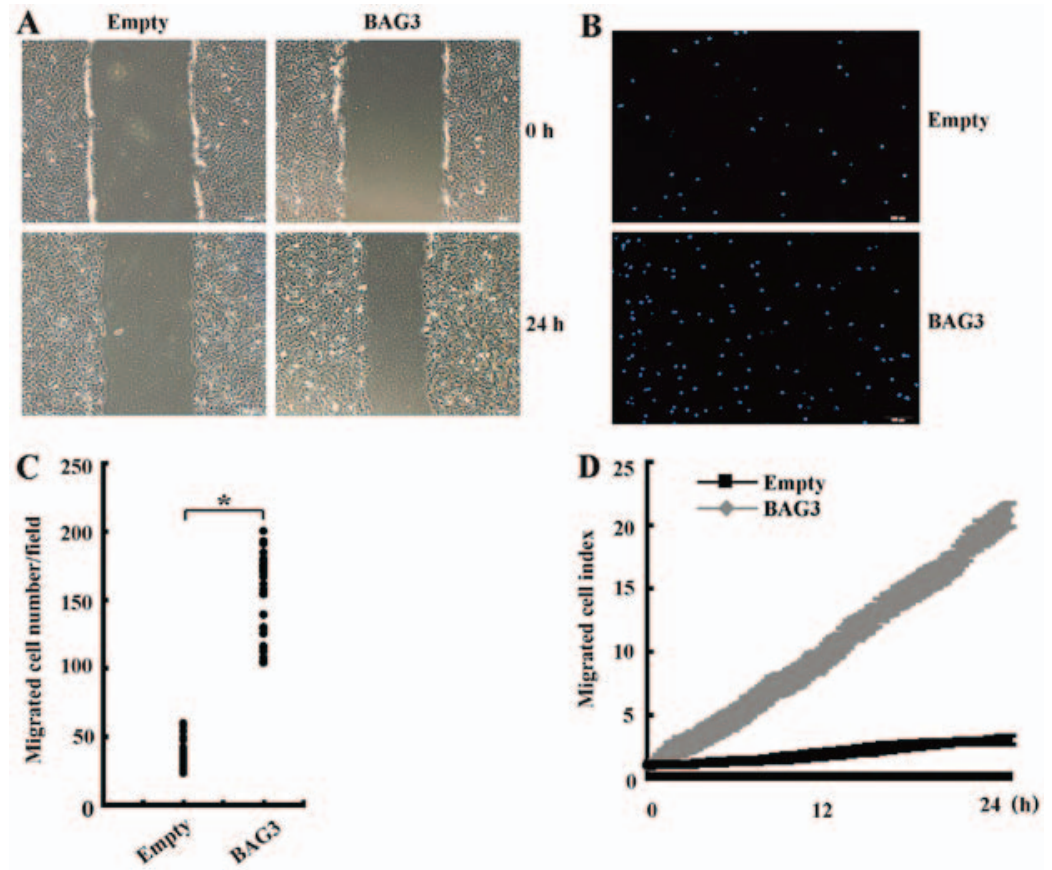

Figure 3. Effects of Bcl-2-associated athanogene 3 (BAG3) on the migration of primary rat vascular smooth muscle cells (VSMCs). (A) Migrated cells were detected by wound healing assay. (B) Migrated cells were stained by Hoechst 33342 in Transwell assay and (C) migrated cells were quantified by ImageJ. (D) Cell migration was monitored continuously by RTCA. The experiments were all repeated three times with reproducible results. "p $<0.05$ compared with the control. 

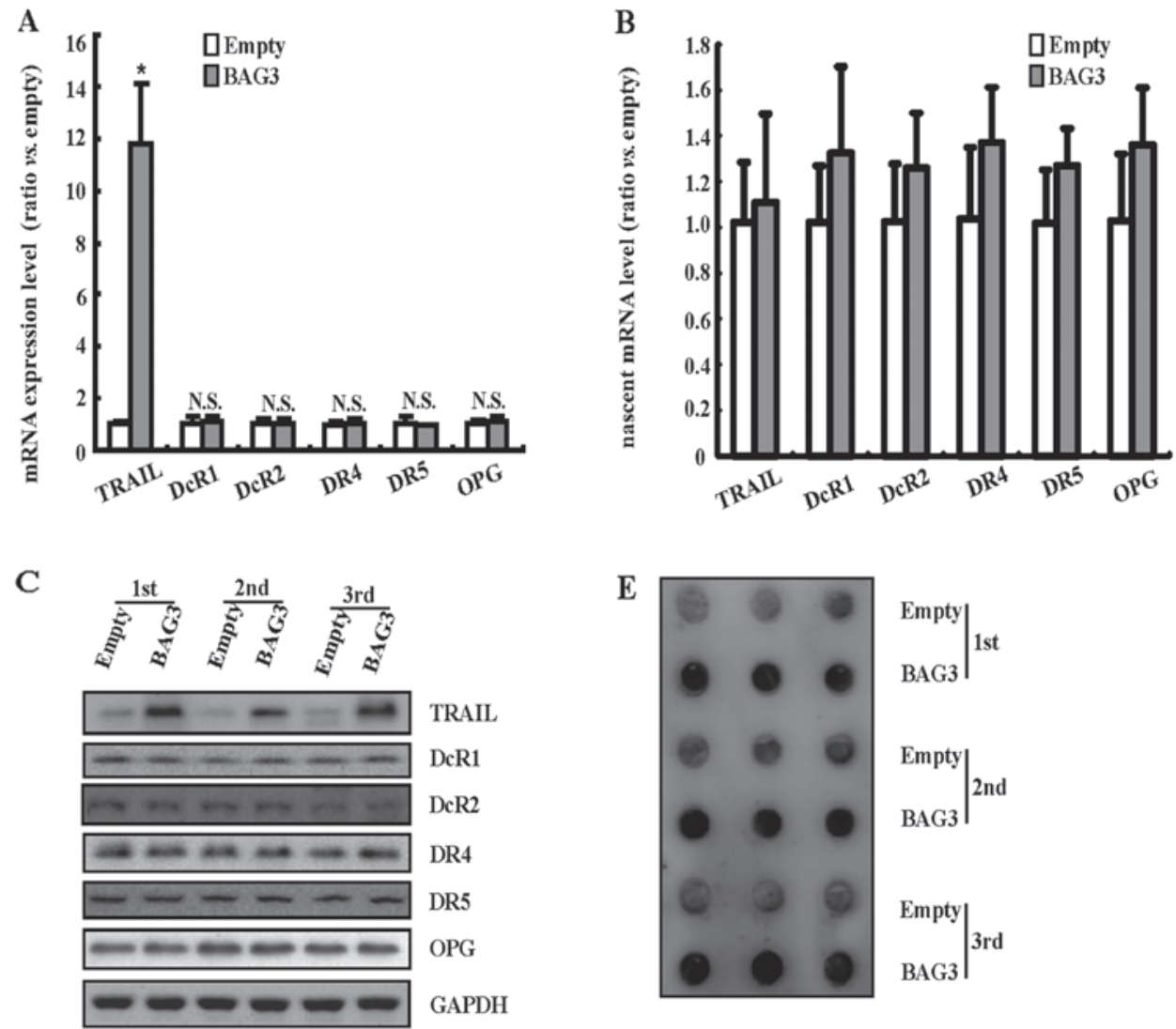

D

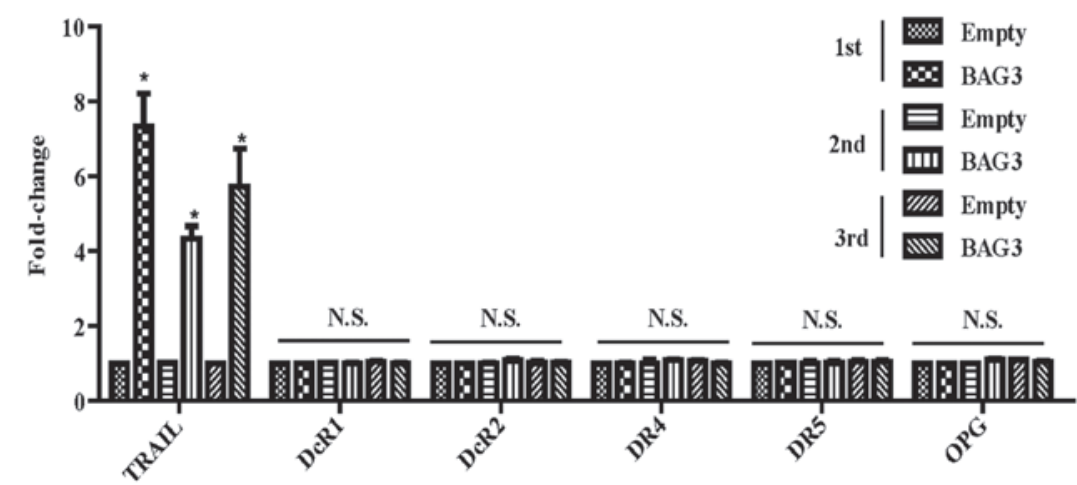

Figure 4. Tumor necrosis factor-related apoptosis inducing ligand (TRAIL) was implicated in the proliferation mediated by Bcl-2-associated athanogene 3 (BAG3) in primary rat vascular smooth muscle cells (VSMCs). (A and B) The total and newly synthesized mRNA of TRAIL and its receptors were detected by RT-PCR. (C) The protein expression of TRAIL and its receptors were detected by western blotting and (D) quantified by ImageJ, GAPDH served as a control. (E) The secreted protein of BAG3 in the cultured media was detected by dot blot. The experiments were all repeated three times with reproducible results. "p $<0.05$ compared with the control.

counting demonstrated that BAG3 overexpression could significantly promote the proliferation of VSMCs, and this biological process was attenuated by TRAIL Ab (Fig. 5A). EdU staining also confirmed that TRAIL Ab could decrease EdU incorporation rate of VSMCs induced by BAG3 overexpression (Fig. 5B). To further investigate the involvement of TRAIL, we generated lentiviral vectors containing shRNAs against TRAIL (shTRAIL) to knock down TRAIL expression in primary rat VSMCs. RT-PCR demonstrated that three shRNAs (shTRAIL\#2, shTRAIL\#4 and shTRAIL\#5) significantly decreased the mRNA level of TRAIL in VSMCs (Fig. 5C). Western blotting demonstrated that shTRAIL\#2, shTRAIL\#4 and shTRAIL\#5 significantly decreased the protein expression of TRAIL in
VSMCs (Fig. 5D and E). Dot blot exhibited that shTRAIL\#2, shTRAIL\#4 and shTRAIL\#5 significantly decreased the secreted protein of TRAIL in culture medium (Fig. 5F). Importantly, cell counting (Fig. 5G) and EdU staining (Fig. 5H) consistently demonstrated that shTAIL\#2, shTRAIL\#4 and shTRAIL\#5 could decrease the proliferation of VSMCs.

Knockdown of TRAIL exerted no obvious influence on the migration of primary rat VSMCs. We then investigated the potential involvement of TRAIL in the migration of primary rat VSMCs. Wound healing assay (Fig. 6A) demonstrated that knockdown of TRAIL per se exerted no obvious influence on the migration of VSMCs. Transwell assay also confirmed that the number of migrated cells did not have significant 

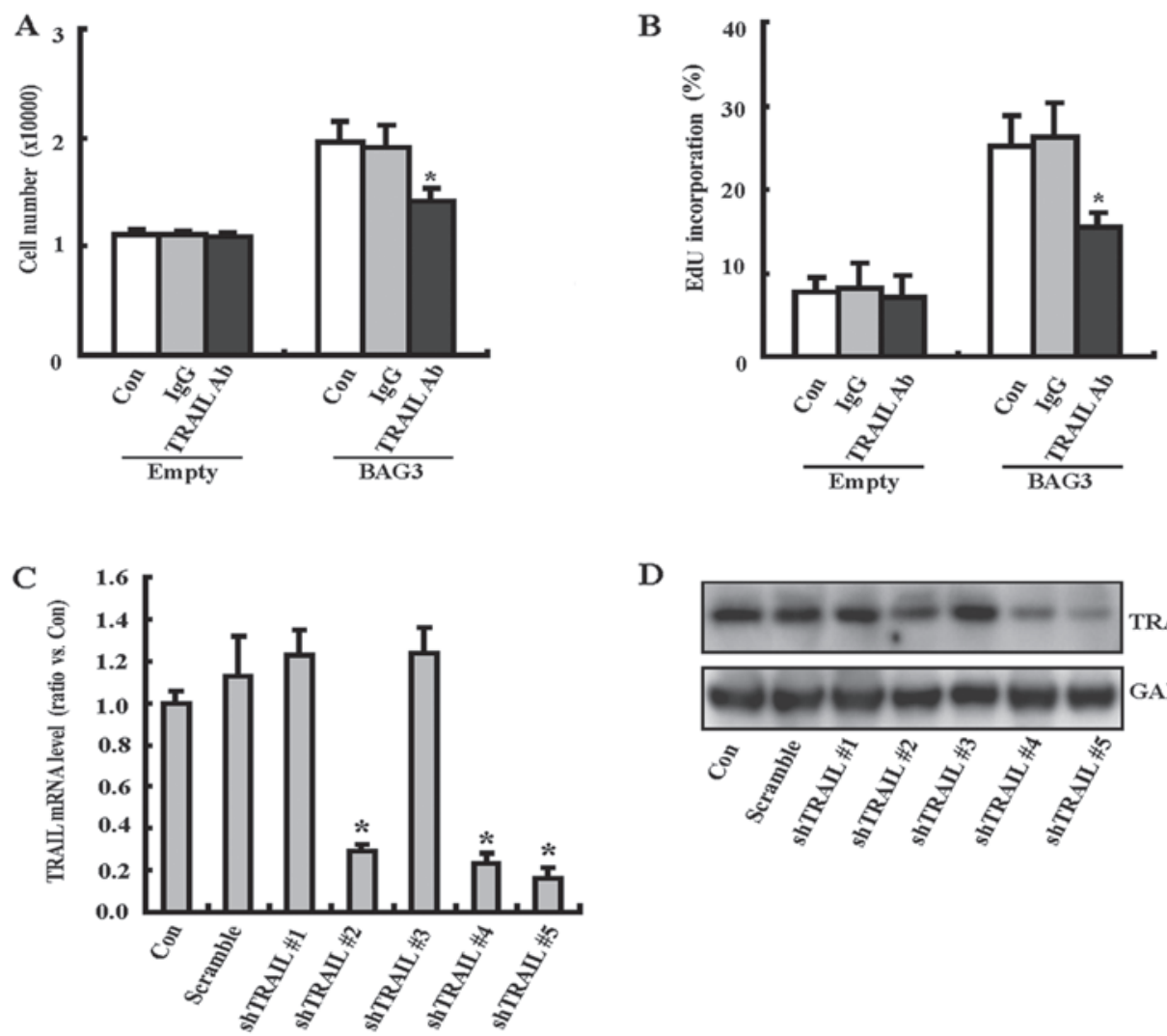

$\mathbf{D}$

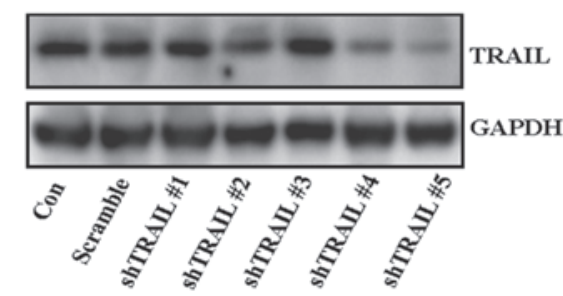

$\mathbf{E}$

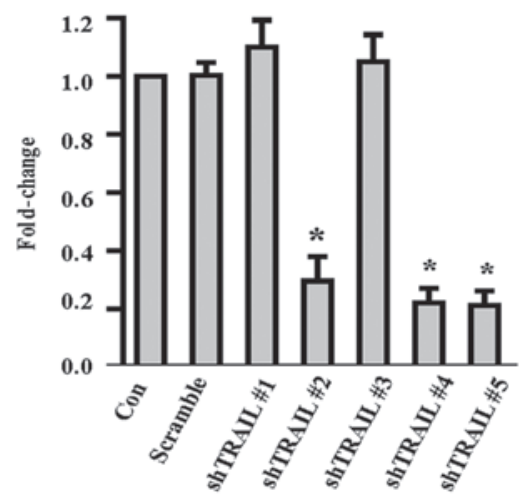

F
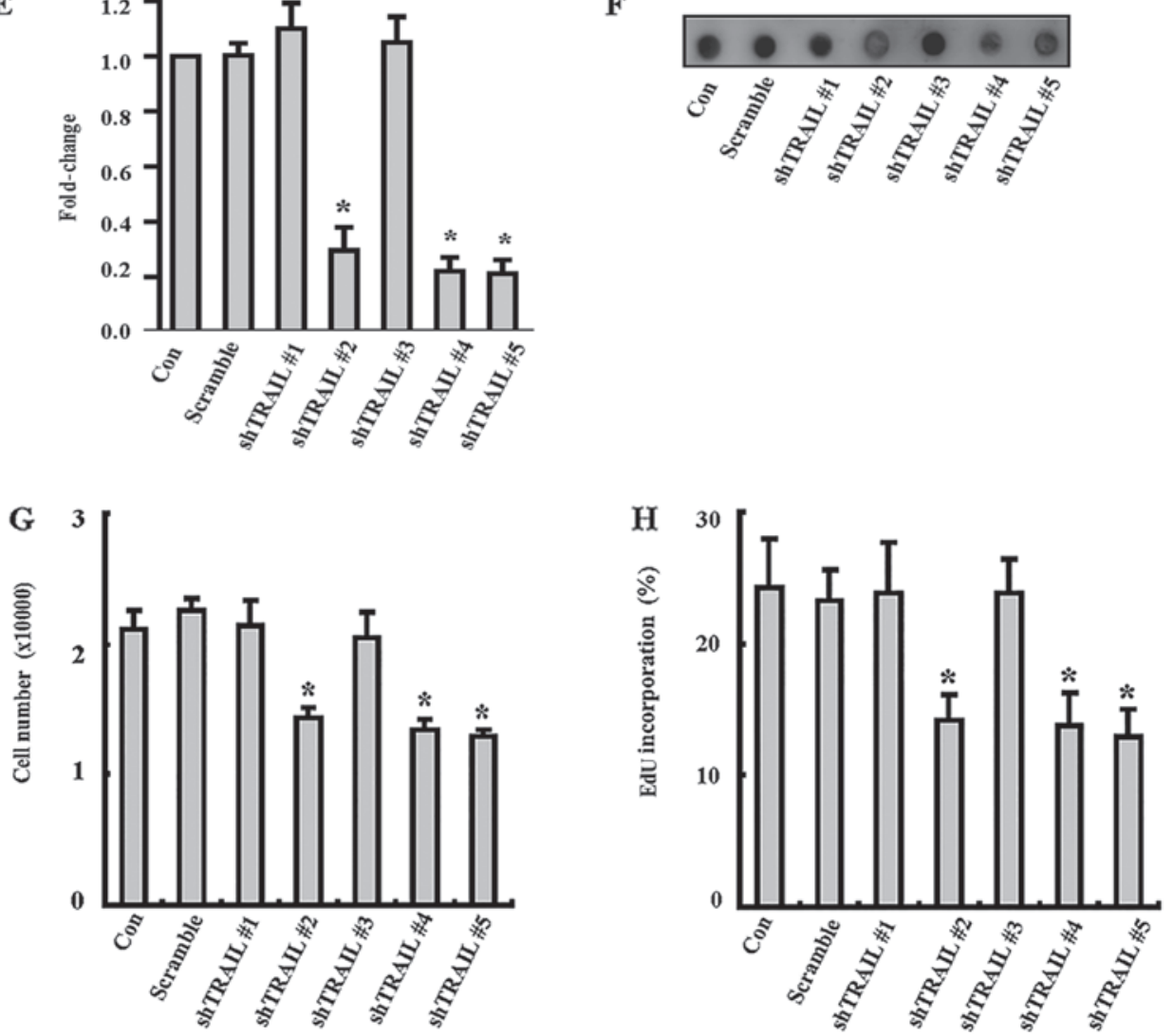

Figure 5. Bcl-2-associated athanogene 3 (BAG3) promotes the proliferation of primary rat vascular smooth muscle cells (VSMCs) via tumor necrosis factor-related apoptosis inducing ligand (TRAIL). Neutralizing antibody against TRAIL (TRAIL Ab) was used to explore the effects of TRAIL on the BAG3-promoted proliferation of VSMCs. After treated with TRAIL Ab, (A) cell viability and (B) EdU incorporation were assessed. We generated lentiviral vectors containing shRNAs against TRAIL (shTRAIL) to knock down TRAIL expression in primary rat VSMCs. (C) The mRNA of TRAIL was detected by RT-PCR. (D) The protein expression of TRAIL were detected by western blotting and quantitated by (E) ImageJ, GAPDH served as a control. (F) The secreted protein of TRAIL in the cultured media was detected by dot blot. (G) Cell proliferation was detected by cell counting and (H) EdU staining. The experiments were all repeated three times with reproducible results. " $\mathrm{p}<0.05$ compared with the control. 

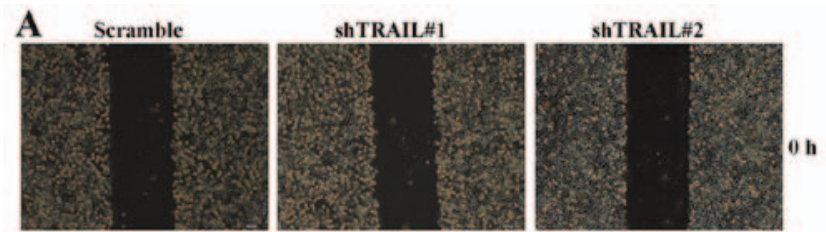

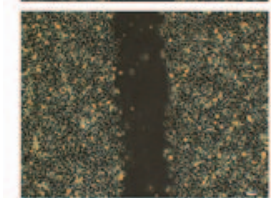

ShTRAIL\#3
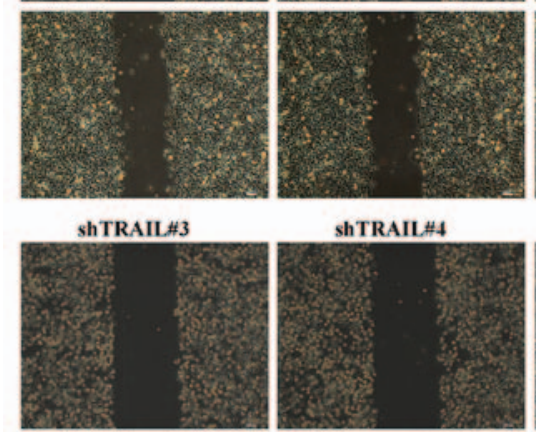

ShTRAIL\#4
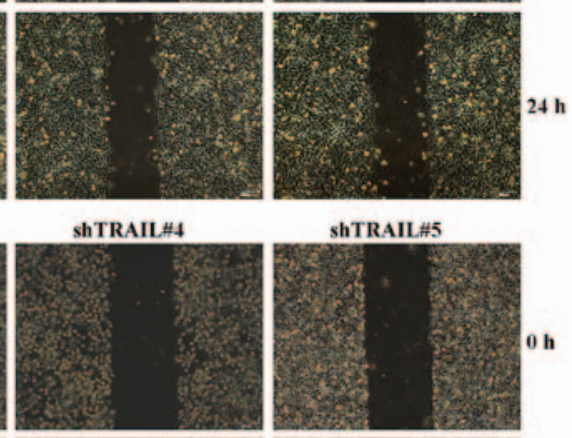

ShTRAIL \#5
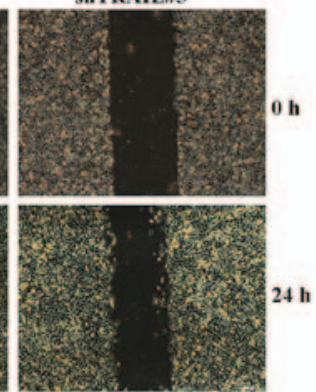

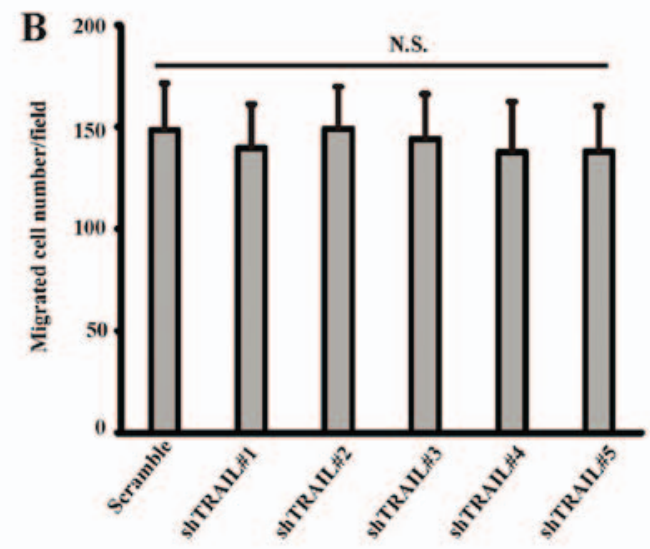

Figure 6. The effect of TRAIL-knockdown on the migration of primary rat vascular smooth muscle cells (VSMCs). We generated lentiviral vectors containing shRNAs against TRAIL (shTRAIL) to knock down TRAIL expression in primary rat VSMCs. (A) Cell migration was detected by wound healing assay and (B) Transwell. The experiments were all repeated three times with reproducible results. * $\mathrm{p}<0.05$ compared with the control.

statistical differences between knockdown group and control group (Fig. 6B).

\section{Discussion}

The present study demonstrated a novel role for BAG3 in regulating the phenotypic transformation in primary rat VSMCs. We found that BAG3 expression increased with continued passages in cultured primary rat VSMCs and promoted the proliferation and migration of primary rat VSMCs. Furthermore, we demonstrated that BAG3 promoted the proliferation of primary rat VSMCs via TRAIL.

Several lines of evidence suggested that the expression of BAG3 was elevated in various tumors including glioblastoma, acute lymphoblastic leukemia and prostate carcinoma (25-27). However, normal tissues seldom express BAG3, except for cardiomyocytes and skeletal muscle cells (14). The expression of BAG3 can be induced by a variety of stimuli such as the early growth response gene-1 (Egr-1) (28), heat shock factor 1 (HSF1) (29), proteasome inhibitors (30), heavy metals as well as heat stress $(31,32)$. Previous studies indicated that BAG3 is involved in various CVD such as myocardial hypertrophy, dilated cardiomyopathy, Takotsubo cardiomyopathy and chronic heart failure (10-13). In the present study, we first confirmed that the expression of BAG3 increased with continued passages in cultured primary rat VSMCs.

In this study, we also demonstrated for the first time that overexpression of BAG3 could promote the proliferation and migration of primary rat VSMCs. BAG3 contains three main protein binding motifs that can mediate potential interactions with chaperons and/or other proteins, a WW domain at the $\mathrm{N}$-terminal, a proline-rich region (PXXP) in the central region, two IPV motifs (Ile-Pro-Val) between the WW domain and the PXXP region (33). Previous study found that in Cos7 cells,
BAG3 could promote the movement and adhesion by binding to the PPDY structure of the guanine nucleotide exchange factor 2 (PDZ-GEF2) through WW domain (34). In MDA435 human breast cancer cells, BAG3 overexpression contributed to the decrease of migration, which is related to PXXP domain (35). Meng et al reported silence of BAG3 can elevate migratory capacity by activating the transcription of ZEB1 in thyroid cancer cells (36). We speculated BAG3 may play different roles in different cells. It is well known that cancer cells are characterized by infinite proliferation, migration and invasion, and similar changes occurred in VSMCs during the formation of atheromatous plaque. Therefore, we hypothesized BAG3 may be a common signaling molecule of the two pathological processes. However, further efforts are still required to elucidate which domain played the main role.

Both BAG3 and TRAIL are involved in apoptosis and immune responses to human immunodeficiency virus (HIV)-1. Existing evidence showed that BAG3 acted synergistically with Bcl-2 and Bax in antagonizing the intrinsic apoptosis induced by TRAIL. In the host cell of HIV-1 infection, BAG3 and $\mathrm{Bcl}-2$ resist apoptosis induced by TRAIL together, which may be an important factor for the chronicity of HIV infection (37,38). Furthermore, Chiappetta et al demonstrated silencing BAG3 could downgrade the apoptotic response to TRAIL in human neoplastic thyroid cells (19). However, the association between BAG3 and TRAIL and their role in the phenotypic transformation of VSMCs has not been fully clarified. The present study demonstrated for the first time that BAG3 upregulation could promote the expression of TRAIL in primary rat VSMCs, which may be realized by decreasing the degradation of TRAIL mRNA. Furthermore, the present study also demonstrated BAG3 promotes the proliferation of primary rat VSMCs via TRAIL. BAG3 is an upstream signalregulated molecule of TRAIL. We originally predicted that 
knockdown of TRAIL may affect BAG3-induced migration. However, no effect was observed in migration assay, which indicated that other signal-regulated molecules may exist.

The present study demonstrated for the first time that BAG3 promotes the proliferation and migration in primary rat VSMCs. BAG3 promoted VSMCs proliferation via the expression of TRAIL. The result revealed incomplete mechanism of atherosclerotic plaque and provided a possible drug target.

\section{Acknowledgements}

Not applicable.

\section{Funding}

This study was supported by National Natural Science Foundation of China (grant nos. 81470417 and 81670231).

\section{Availability of data and material}

The datasets used and/or analyzed during the current study are available from the corresponding author on reasonable request.

\section{Authors' contributions}

YF and YS made substantial contributions to conception and design. YF, YeC, SC, YL, YiC, GS, SY, NY and CL cooperated with each other to perform the experiments in this manuscript. YF and YeC were major contributors in writing the manuscript. YS gave the final approval of the version to be published. All authors read and approved the final manuscript.

\section{Ethics approval and consent to participate}

Not applicable.

\section{Consent for publication}

Not applicable.

\section{Competing interests}

The authors declare that they have no competing interests.

\section{References}

1. Doran AC, Meller N and McNamara CA: Role of smooth muscle cells in the initiation and early progression of atherosclerosis. Arterioscler Thromb Vasc Biol 28: 812-819, 2008.

2. Rzucidlo EM, Martin KA and Powell RJ: Regulation of vascular smooth muscle cell differentiation. J Vasc Surg 45 (Suppl A) 25-32, 2007

3. Owens GK: Regulation of differentiation of vascular smooth muscle cells. Physiol Rev 75: 487-517, 1995.

4. Owens GK, Kumar MS and Wamhoff BR: Molecular regulation of vascular smooth muscle cell differentiation in development and disease. Physiol Rev 84: 767-801, 2004.

5. Orr AW, Hastings NE, Blackman BR and Wamhoff BR: Complex regulation and function of the inflammatory smooth muscle cell phenotype in atherosclerosis. J Vasc Res 47: 168-180, 2010.

6. Iwasaki M, Homma S, Hishiya A, Dolezal SJ, Reed JC and Takayama S: BAG3 regulates motility and adhesion of epithelial cancer cells. Cancer Res 67: 10252-10259, 2007.
7. Bloemberg D, McDonald E, Dulay D and Quadrilatero J: Autophagy is altered in skeletal and cardiac muscle of spontaneously hypertensive rats. Acta Physiol (Oxf) 210: 381-391, 2014

8. Liu BQ, Du ZX, Zong ZH, Li C, Li N, Zhang Q, Kong DH and Wang HQ: BAG3-dependent noncanonical autophagy induced by proteasome inhibition in HepG2 cells. Autophagy 9: 905-916, 2013.

9. Rosati A, Graziano V, De Laurenzi V, Pascale M and Turco MC: BAG3: a multifaceted protein that regulates major cell pathways. Cell Death Dis 2: e141, 2011.

10. Falco A, Festa M, Basile A, Rosati A, Pascale M, Florenzano F, Nori SL, Nicolin V, Di Benedetto M, Vecchione ML, et al: BAG3 controls angiogenesis through regulation of ERK phosphorylation. Oncogene 31: 5153-5161, 2012.

11. Norton N, Li D, Rieder MJ, Siegfried JD, Rampersaud E, Züchner S, Mangos S, Gonzalez-Quintana J, Wang L, McGee S, et al: Genome-wide studies of copy number variation and exome sequencing identify rare variants in BAG3 as a cause of dilated cardiomyopathy. Am J Hum Genet 88: 273-282, 2011.

12. Citro R, d'Avenia M, De Marco M, Giudice R, Mirra M, Ravera A, Silverio A, Farina R, Silvestri F, Gravina P, et al: Polymorphisms of the antiapoptotic protein bag3 may play a role in the pathogenesis of tako-tsubo cardiomyopathy. Int $\mathrm{J}$ Cardiol 168: 1663-1665, 2013.

13. De Marco M, Falco A, Basile A, Rosati A, Festa M, d'Avenia M, Pascale M, Dal Piaz F, Bisogni R, Barcaroli D, et al: Detection of soluble BAG3 and anti-BAG3 antibodies in patients with chronic heart failure. Cell Death Dis 4: e495, 2013.

14. Hishiya A, Kitazawa T and Takayama S: BAG3 and Hsc70 interact with actin capping protein CapZ to maintain myofibrillar integrity under mechanical stress. Circ Res 107: 1220-1231, 2010.

15. Wiley SR, Schooley K, Smolak PJ, Din WS, Huang CP, Nicholl JK, Sutherland GR, Smith TD, Rauch C, Smith CA, et al: Identification and characterization of a new member of the TNF family that induces apoptosis. Immunity 3: 673-682, 1995.

16. Pan G, O'Rourke K, Chinnaiyan AM, Gentz R, Ebner R, Ni J and Dixit VM: The receptor for the cytotoxic ligand TRAIL. Science 276: 111-113, 1997.

17. Pan G, Ni J, Wei YF, Yu G, Gentz R and Dixit VM: An antagonist decoy receptor and a death domain-containing receptor for TRAIL. Science 277: 815-818, 1997.

18. Emery JG, McDonnell P, Burke MB, Deen KC, Lyn S, Silverman C, Dul E, Appelbaum ER, Eichman C, DiPrinzio R, et al: Osteoprotegerin is a receptor for the cytotoxic ligand TRAIL. J Biol Chem 273: 14363-14367, 1998.

19. Chiappetta G, Ammirante M, Basile A, Rosati A, Festa M, Monaco M, Vuttariello E, Pasquinelli R, Arra C, Zerilli M, et al: The antiapoptotic protein BAG3 is expressed in thyroid carcinomas and modulates apoptosis mediated by tumor necrosis factor-related apoptosis-inducing ligand. J Clin Endocrinol Metab 92: 1159-1163, 2007.

20. Ma M, Guo X, Chang Y, Li C, Meng X, Li S, Du ZX, Wang HQ and Sun Y: Advanced glycation end products promote proliferation and suppress autophagy via reduction of cathepsin D in rat vascular smooth muscle cells. Mol Cell Biochem 403: 73-83, 2015.

21. Salabei JK, Cummins TD, Singh M, Jones SP, Bhatnagar A and Hill BG: PDGF-mediated autophagy regulates vascular smooth muscle cell phenotype and resistance to oxidative stress. Biochem J 451: 375-388, 2013.

22. Chang Y, Li Y, Ye N, Guo X, Li Z, Sun G and Sun Y: Atorvastatin inhibits the apoptosis of human umbilical vein endothelial cells induced by angiotensin II via the lysosomal-mitochondrial axis. Apoptosis 21: 977-996, 2016.

23. Ke N, Wang X, Xu X and Abassi YA: The xCELLigence system for real-time and label-free monitoring of cell viability. Methods Mol Biol 740: 33-43, 2011.

24. Chen S, Liu B, Kong D, Li S, Li C, Wang H and Sun Y: Atorvastatin calcium inhibits phenotypic modulation of PDGF-BB-induced VSMCs via down-regulation the Akt signaling pathway. PLoS One 10: e0122577, 2015.

25. Gentilella A and Khalili K: BAG3 expression in glioblastoma cells promotes accumulation of ubiquitinated clients in an Hsp70-dependent manner. J Biol Chem 286: 9205-9215, 2011.

26. Zhu H, Wu W, Fu Y, Shen W, Miao K, Hong M, Xu W, Young KH, Liu P and Li J: Overexpressed BAG3 is a potential therapeutic target in chronic lymphocytic leukemia. Ann Hematol 93: 425-435, 2014.

27. Staibano S, Mascolo M, Di Benedetto M, Vecchione ML, Ilardi G, Di Lorenzo G, Autorino R, Salerno V, Morena A, Rocco A, et al: BAG3 protein delocalisation in prostate carcinoma. Tumour Biol 31: 461-469, 2010. 
28. Gentilella A, Passiatore G, Deshmane S, Turco MC and Khalili K: Activation of BAG3 by Egr-1 in response to FGF-2 in neuroblastoma cells. Oncogene 27: 5011-5018, 2008

29. Franceschelli S, Rosati A, Lerose R, De Nicola S, Turco MC and Pascale M: Bag3 gene expression is regulated by heat shock factor 1. J Cell Physiol 215: 575-577, 2008.

30. Du ZX, Meng X, Zhang HY, Guan Y and Wang HQ: Caspase-dependent cleavage of BAG3 in proteasome inhibitors-induced apoptosis in thyroid cancer cells. Biochem Biophys Res Commun 369: 894-898, 2008.

31. Liao Q, Ozawa F, Friess H, Zimmermann A, Takayama S, Reed JC, Kleeff J and Büchler MW: The anti-apoptotic protein BAG-3 is overexpressed in pancreatic cancer and induced by heat stress in pancreatic cancer cell lines. FEBS Lett 503: 151-157, 2001.

32. Pagliuca MG, Lerose R, Cigliano S and Leone A: Regulation by heavy metals and temperature of the human BAG-3 gene, a modulator of Hsp70 activity. FEBS Lett 541: 11-15, 2003.

33. Gamerdinger M, Carra S and Behl C: Emerging roles of molecular chaperones and co-chaperones in selective autophagy: focus on BAG proteins. J Mol Med (Berl) 89: 1175-1182, 2011.
34. Iwasaki M, Tanaka R, Hishiya A, Homma S, Reed JC and Takayama S: BAG3 directly associates with guanine nucleotide exchange factor of Rap1, PDZGEF2, and regulates cell adhesion. Biochem Biophys Res Commun 400: 413-418, 2010.

35. Kassis JN, Virador VM, Guancial EA, Kimm D, Ho AS Mishra M, Chuang EY, Cook J, Gius D and Kohn EC: Genomic and phenotypic analysis reveals a key role for CCN1 (CYR61) in BAG3-modulated adhesion and invasion. J Pathol 218: 495-504, 2009.

36. Meng X, Kong DH, Li N, Zong ZH, Liu BQ, Du ZX, Guan Y, Cao L and Wang HQ: Knockdown of BAG3 induces epithelial-mesenchymal transition in thyroid cancer cells through ZEB1 activation. Cell Death Dis 5: e1092, 2014.

37. Huang Y, Erdmann N, Zhao J and Zheng J: The signaling and apoptotic effects of TNF-related apoptosis-inducing ligand in HIV-1 associated dementia. Neurotox Res 8: 135-148, 2005.

38. Cummins NW and Badley AD: Mechanisms of HIV-associated lymphocyte apoptosis: 2010. Cell Death Dis 1: e99, 2010. 